\title{
MS11-P05 | PRESTO INTEGRATIVE STRUCTURE BIOLOgY SOFTWARE FOR ALL PLATFORMS
}

Unge, Johan (MAX IV, Lund University, Lund, SWE)

Objective

With the larger amount of data generated in lesser time the need for better resources to share and analyse the data is increasing. Projects tend to span over several techniques to answer the scientific questions leading to Integrative Structure Biology. The need to be able to share the results, software and competence across the fields is accelerating. The software project PReSTO has been initiated with the aim of providing a common software package for all technique's analysis of structure biology data on local, remote, single and multimode platforms, with the emphasis of High-Performance Computing infrastructures.

Result

Protein Science Facility (PSF) from Karolinska Institute in Stockholm and National Supercomputer Centre (NSC) in Linköping, the Swedish synchrotron light source MAX IV and Lunarc, the supercomputer centre in Lund, Sweden, runs PRESTO to support Swedish structural biologist with powerful analysing tools and an interface to the data generated to MAX IV. PReSTO can run on any machine and is especially designed to be make an efficient use of the High-Performance Computing facilities using several nodes in an user friendly way. It supports a virtual desktop with menu based support for parallel processing and batch or interactive processing of data.

Future

The project has funding to invite all parties of Integrative Structural Biology including Neutron MX (NMX), Nuclear Magnetic Resonance (NMR) and cryo-electron microscopy (CryoEM). The techniques used can be ported to any user, institution of facility around Europe. 